\title{
Problématique des agglomérations suisses
}

\section{Zusammenfassung}

Dieser Artikel gibt einen Überblick über die wichtigsten Fragen, die sich heute in der Agglomeration der Schweiz stellen. Er analysiert die städtische Entwicklungspolitik, die Verkehrspolitik, die Verkehrsplanung sowie die Sozialfrage. Unsere Studie betrifft sechs Städte der deutschen und französischen Schweiz. Es hat sich u. a. bestätigt, daß besonders die Verkehrspolitik sehr unterschiedlich angegangen wird: in der deutschen Schweiz ist sie weiter entwickelt.

\section{Introduction}

Lobjet de cet article est tiré des préoccupations soulevées par le programme national de recherche, «Ville et transport» - PNR 25 -, financé par le fonds national de la recherche suisse entre 1990 et 1991 . Nous tenterons de définir les problèmes urbains qui se posent d'une façon souvent récurrente dans plusieurs agglomérations.'

Nous pouvons illustrer notre propos par un seul exemple que démontre a fortiori les relations complexes qui se développent dans l'ensemble des agglomérations. Autour de la dichotomie ville/centre et communes suburbaines ou périphériques viennent se greffer d'autres problèmes touchant à la gestion intercommunale, à la péréquation fiscale entre lieu de travail et lieu de résidence. Les enjeux d'aménagement et de politique économique globale sont occultés par une concurrence, une lutte économique entre communes pour accroître leurs rentrées financières et donc leurs possibilités de réaliser des équipements divers, répondant à des besoins propres souvent au bénéfice de leurs habitants et à la limite au profit d'une équipe politique soutenue par des citoyens/électeurs. Ceci au grand dam de l'intérêt régional bien compris. Au niveau d'une ville, d'une commune, des questions se posent quant à savoir comment s'organise l'aménagement des quartiers, quels sont les priorités et de quels moyens dispose-t-on pour concrétiser le développement socio-économique de nos quartiers. C'est pour cette raison qu'il nous faut analyser nos agglomérations.

\section{Les problèmes urbains}

\subsection{Introduction}

La complexification des modes de production et leurs incidences interdépendantes dans un champs d'analyse toujours plus vaste - démographique, sociologique, géographique, économique, juridique et politique -, nous impose de retracer très rapidement les étapes de la traduction concrète de ce mécanisme au niveau du phénomène urbain.

La Suisse, à l'image des pays européens, a connu de profondes mutations de son tissu urbain. L'histoire de l'urbanisation en Suisse est riche de phases fort diverses au cours des derniers siècles, et pour s'en rendre compte des ouvrages font référence en la matière. ${ }^{2}$ Nous ne reprendrons que les éléments les plus récents, à partir du moment où l'état de type ancien régime se modernise avec la création de l'Etat fédéral en 1848. Ce qui correspond à une profonde évolution du système économique -Révolution industrielle-, entraînant une remise en cause des structures sociales et politiques de l'état. Cette première phase qui dure jusqu'à la Seconde Guerre mondiale se manifeste par un renforcement des villes sous la poussée de l'industrie et de la croissance démographique urbaine qui touche surtout les villes centre, au détriment des villages qui sont peu touchés par les bouleversements économiques.

La phase qui correspond aux "Trente Glorieuses» de la fin du dernier conflit mondial au début des années septante avec la fameuse crise économique qui secoua non seulement notre croyance aux progrès linéaires mais également la certitude de voir croître indéfiniment nos villes, est caractérisée par une croissance urbaine rapide et soutenue, induite par une économie en plein essor et par une augmentation notoire de la population urbaine. Nous pouvons parler d'essor urbain généralisé dans lequel deux tendances sont nettes. La première est la poursuite de l'urbanisation par densification de l'espace urbain; la seconde est liée à la suburbanisation favorisée par une motorisation importante de la population et la disponibilité de terrains à proximité des villes.

La phase actuelle est largement tributaire de deux paramètres. Le premier est dû à l'éclatement de la crise économique en 1974, qui a remodelé le visage de la croissance économique. Le second est plus intéressant au niveau de la réflexion urbanistique, il s'agit d'une tendance qui se manifeste de plus en plus nettement, à savoir un renverse-

Jean-Pierre Wolff, Dr, Université de Neuchâtel, Institut de Géographie/Université d'Angers (France), Dépt. de Géographie. 
ment d'évolution avec un recul de la population des villes-centres et ceci au profit des communes suburbaines puis périurbaines. La crise économique qui plonge les pays industrialisés dans une récession marquée, se traduit au niveau de l'urbanisation par un abandon de projets ou tout du moins d'un report pour des jours plus favorables. Et ceci est notoire dans les centres urbains et les villes-centres qui accueillaient ce type d'équipements sièges de société, de banques, centres de recherche, palais des congrès, grands hôtels, etc. Ce ralentissement économique se double par le développement d'un sentiment écologisant qui prône un retour à la nature et donc à la mort des cités. Ce «besoin écologiste» profite d'une certaine disponibilité en terrains et ceci concourt au renforcement de la périurbanisation.

Enfin nous pouvons définir avec beaucoup de prudence ce qui se passe à la fin de la décennie quatre-vingt et au début des années nonante et qui correspond à une reprise de l'urbanisation, comparable en certains points à celle de l'avant-crise, mais caractérisée par le découplage du phénomène croissance démographique/extension de l'urbanisation. Nous observons la reprise du city druck, la construction de centres directionnels, la poursuite des programmes liés à la construction d'infrastructures pour les transports tant privés que publics. Mais en même temps, on observe un changement de logique dans cette urbanisation car on ne baigne plus dans la même euphorie inconsciente de l'avant-crise. Le premier est lié aux prévisions d'évolution qui sont fortement minorées et ceci se lit dans les plans d'urbanisme, dans les schémas de développement des équipements lourds ou structurants - centres commerciaux en périphérie, grands complexes sportifs et culturels, etc. Le temps de toutes les audaces semble révolu et simultanément une nouvelle préoccupation s'érige en politique urbaine, à savoir la qualité de la vie. Au nom de ce thème on assiste à un retour vers le centre-ville qui retrouve une valeur patrimoniale considérable relevant d'intérêts immobiliers bien précis. Cette reconquête des centres se fait parallèlement à la continuation du phénomène de périurbanisation qui tend à engendrer une ségrégation sociale de plus en plus nette entre ville-centre, entre quartiers et entre communes suburbaines.

Est-ce la fin du gigantisme remplacé par l'émergence d'une nouvelle exigence sociale et écologiste ou une recomposition spatialo-sociale non dénuée d'intérêts économiques bien compris?

\subsection{Les problèmes présents}

Les nombreux problèmes qui se posent sont induits par les réalités socio-économiques et se traduisent au niveau d'un cadre spatial continuellement déstructuré/restructuré et d'une société hétérogène qui est soumise à cette évolution globale. L'urbanisation qui se met en place est la conséquence des intérêts économiques en présence et de la structure juridique qui permet son inscription tant dans l'espace que dans les réglementations urbanisti- ques. Comme en Suisse le fédéralisme et l'autonomie des communes définissent des structures administratives et politiques bien différentes d'un canton à l'autre, nous pourrions espérer repérer des situations bien typées. Cette hypothèse est à remettre en question quant à la logique de l'urbanisation, car celle-ci est soutenue par l'économie. On peut percevoir les mêmes grands problèmes dans toutes nos agglomérations - pression immobilière, transports, pollution, question sociale...

Nous venons d'employer plusieurs termes qui renvoient à des notions soit spatiales, soit institutionnelles, soit enfin socio-économiques et nous devons, afin que la poursuite de notre travail aille de soi, nous entendre sur ces termes en précisant leur sens.

L'agglomération, terme générique, renvoie à différents auteurs. ${ }^{3}$ "Le terme d'agglomération traduit les transformations profondes qui sont liées à l'urbanisation généralisée, au développement des transports modernes et à l'apparition des centres commerciaux ou de centres directionnels à la périphérie des cités les plus importantes. Le terme convient bien pour saisir une réalité où les formes sont moins clairement ordonnées que par le passé: il traduit la généralisation d'espaces suburbain, souvent très monotones, et où il est difficile de lire dans les paysages une organisation claire: seule l'analyse des flux et des espaces d'activité en fait comprendre la vie». ${ }^{4}$ La notion de commune est cruciale au niveau institutionnel. C'est une collectivité territoriale dont l'étendue sert à la fois d'assise à une circonscription de l'Etat -du canton- et à une collectivité locale. La commune est donc une entité spatiale et une personne morale ou juridique. Sur le plan statistique elle peut être considérée comme rurale ou urbaine en fonction de sa population. Suivant les cantons, l'autonomie communale varie beaucoup, c'est ainsi que dans le canton de Genève celle-ci est des plus limitée, par contre dans celui de Vaud, elle est encore très marquée. Néanmoins une évolution récente tend à limiter cette autonomie en matière d'aménagement urbain et ceci au profit d'une politique planificatrice globale et mise en place par les cantons. En général cette forte autonomie communale est un frein à une politique volontariste et globalisante en matière d'aménagement régional. Des projets de localisation, de construction d'équipements publics, d'infrastructures routières ou de politique globale des transports ne peuvent pas aboutir par défaut d'une volonté politique commune. Les égoïsmes locaux sont dénoncés dans plusieurs agglomérations, la commune-centre accuse les communes périphériques de profiter des équipements lourds mis en place sur son territoire et de ne pas participer suffisamment à la prise en charge des coûts d'investissement et de gestion. Par contre les communes périphériques dénoncent l'impérialisme de la commune-centre en matière de politique d'aménagement régional. Donc certains des problèmes relevés transcendent les limites communales - une pénétrante urbaine, une salle de spectacle à vocation régionale, etc. - et cristallisent des oppositions intercommunales avant de déboucher si les possibilités et les volontés le permettent, sur une réalisation intercommunale. 
Le terme "urbain» caractérise la ville par opposition au monde rural et il a donné naissance au néologisme «urbanisation» qui définit l'action d'urbaniser un espace et la concentration croissante de population et d'activités diverses dans un territoire. Donc l'urbain se rapporte, systématiquement, dans notre étude à la ville par opposition aux termes suivants - suburbain, périurbain, rurbain qui indiquent une avancée, une diffusion de l'urbanisation dans l'espace rural.

Quant au concept «urbanisation» il renvoie à deux sens principaux. Le premier indique l'action d'urbaniser, donc il s'agit d'une transformation architectonique et morphologique d'un espace. Le deuxième sens est pris dans son acceptation sociologique et il définit les mutations de l'ensemble des populations aussi bien citadines que rurales, touchées par la modernisation des formes de pensée, de comportement et en particulier de consommation.

Après avoir apporté quelques points de définition à notre champ d'études, il est logique d'appréhender les grands thèmes transversaux qui s'inscrivent systématiquement ou non dans nos six agglomérations retenues.

\subsection{Une logique d'ingrédients}

Diversité politico-administrative et socio-culturelle, mais homogénéité économique caractérisent le réseau urbain helvétique. L'angle économiciste est fondamental pour analyser cette logique inhérente au phénomène urbain. Cette homogénéité économique ne signifie pas qu'il y ait de partout les mêmes intérêts, les mêmes enjeux économiques; mais que le réseau dans lequel s'insèrent ces agglomérations forme un système capitalistique dans lequel nos agglomérations se situent selon une hiérarchie rigoureuse. Zurich et Genève, au sommet, dominent Bâle, Berne et Lausanne, puis à des échelons inférieurs des villes comme Neuchâtel, Winterthur et La Chaux-deFonds. Ce tableau de positionnement des agglomérations se réduit à une lecture des plus liminaires et dans la réalité il est des plus complexe à appréhender. Néanmoins il nous donne une vision globale de l'importance socio-économique des agglomérations. La Chaux-deFonds apparaît en périphérie du "croissant économique» suisse et cela explique en partie la pugnacité des autorités et des milieux économiques qui défendent leur ville avec la dernière énergie. Tandis que Winterthur est totalement intégré dans le triangle d'or économique, mais se trouve dépendante de Zurich du fait de leur proximité géographique et d'une certaine complémentarité sur le plan économique.

Néanmoins une même logique économique prévaut à toutes ces actions qui s'inscrivent et orientent l'urbanisation. Une politique de gestion des infrastructures ou des équipements urbains ne dépend pas uniquement des besoins de la population ou de la volonté des édiles locaux, mais aussi et surtout de l'économique en tant que force dynamique de notre société. La présence d'un système de transports en commun très bien développé est un atout pour la localisation de certaines entreprises ou centres de recherche et ce surtout dans des villes d'une certaine importance comme Genève ou Berne, mais il est également pris en considération dans les autres agglomérations. Et en renversant notre hypothèse on peut affirmer que pour attirer ou retenir des sociétés, il faut réaliser un réseau de transport public optimal, garant de l'efficacité urbaine. Donc c'est cette logique économiciste qui produit ou destructure la ville pour recomposer nos agglomérations urbaines, interdépendantes les unes des autres, via ce vecteur économique.

Un effet de taille est à introduire dans nos propos. La taille d'une agglomération induit des éléments spécifiques - dimensionnement des équipements, pendularité d'une partie de la population. La Chaux-de-Fonds n'a pas besoin de parkings souterrains aux abords de son centre, vue l'étroitesse de la partie centrale et les disponibilités en places de stationnement à proximité immédiate du centre. Le réseau de transports publics bernois est nettement plus étoffé que celui de Neuchâtel et cela se justifie par des disparités tant spatiales que démographiques, mais cela n'induit pas que des difficultés n'existent pas dans cette dernière ville. Même si l'effet taille est à retenir, il faut surtout s'occuper de la logique à la base du monde urbain, à savoir sa fonctionnalité et donc sa rentabilité économique globale que l'on retrouve dans toutes agglomérations et dans leur hiérarchisation.

\section{Le choix des agglomérations}

Avant d'entrer dans le cœur du sujet à savoir quels sont les grands problèmes des agglomérations retenues pour notre étude, nous nous devons de les mentionner. Il s'agit par ordre alphabétique des agglomérations suivantes: Berne, Genève, La Chaux-de-Fonds, Lausanne, Neuchâtel et Winterthur.

Nos différentes agglomérations sont formées d'un nombre particulier de communes - cf. tableau «Zones urbaines en 1980". C'est ainsi que celle de Genève en comprend 45 , celle de Lausanne 42 , celle de Berne 22, celle de Neuchâtel 11, celle de Winterthur 9 et enfin le cas atypique de La Chaux-de-Fonds dans cette cohorte étudiée. Ceci est dû à la superficie assez vaste du territoire de cette commune et dont la zone urbanisée n'utilise qu'une partie de la commune.

Ces agglomérations présentent une diversité stimulante sur de nombreux plans. Géographiquement, elles sont toutes situées dans le "croissant utile» à savoir le plateau helvétique, à l'exception de La Chaux-de-Fonds localisée dans le Jura à 1000 mètres d'altitude et en position périphérique non seulement au niveau géographique mais également économique par rapport au reste du pays. Economiquement, elles ont toutes été façonnées par l'essor de l'industrialisation au siècle passé et au début de celui-ci; et ensuite par le développement du tertiaire. Cependant des nuances sont à ajouter et qui jouent sur leur urbanisation. Des villes comme La Chaux-de-Fonds, Winterthur sont encore très marquées par leurs activités industrielles - mixité des fonctions habitat/industrie, 


\begin{tabular}{lcrrrrr}
\hline \multirow{2}{*}{ Agglomérations } & Nombre de & \multicolumn{5}{c}{ Population résidente } \\
& communes & 1.12 .1950 & 1.12 .1960 & 1.12 .1970 & 2.12 .1980 & 1.1 .1987 \\
\hline Berne & 22 & 214760 & 252740 & 291597 & 298125 & 298816 \\
La Chaux-de-Fonds & & 33300 & 38906 & 42347 & 37234 & 35726 \\
Genève & 45 & 207417 & 265747 & 342554 & 363953 & 380093 \\
Lausanne & 42 & 152936 & 195775 & 247782 & 253003 & 259908 \\
Neuchâtel & 11 & 44985 & 55343 & 69406 & 66494 & 65852 \\
Winterthur & 9 & 77684 & 94380 & 110041 & 107752 & 107354 \\
\hline
\end{tabular}

image de marque particulière, friches industrielles - et ces deux villes font figure d'industrieuses et de quelque peu défavorisées surtout en ce qui concerne la ville jurassienne. Par contre des cités comme Genève et Berne sont des villes tertiaires par excellence; l'une étant le siège d'une multitude d'activités internationales, l'autre constituant la capitale politique du pays. Quant aux deux dernières de notre recherche, elles sont des villes où activités secondaires et tertiaires sont présentes, même si ce sont les services qui assurent à l'heure actuelle le rôle primordial sur le plan économique. Démographiquement elles ont connu toutes une évolution comparable jusqu'au début des années septante puis à partir des difficultés économiques rencontrées leur croissance s'est plus ou moins arrêtée avant de repartir en introduisant un déphasage entre la ville-centre et les communes les plus périurbaines où les disponibilités en terrains relativement bon marchés étaient présentes.

Le recensement et l'analyse des grands problèmes concernant l'intégralité de notre champs d'études peuvent apparaître comme réducteur et à la limite quelque peu fallacieux. Car les questions soulevées et les solutions prônées sont particulières à chaque cas et si on se réfêre à une étude récente réalisée dans le cadre de l'OEPR $^{5}$ cette affirmation est étayée.

C'est ainsi que les questions liées aux transports et à la circulation sont fort pertinentes à Lausanne. Par contre, le logement et la question foncière et immobilière apparaissent comme vitaux à Genève. Tandis qu'à Neuchâtel les interrogations économiques et fiscales font partie des principales préoccupations des autorités et des milieux concernés. Enfin à Berne deux problèmes sont fort poignants; il s'agit de la question de la drogue, de la réinsertion des drogués et enfin de la crise du logement.

Mais au-delà de ces spécificités locales nous retrouvons d'une façon plus ou moins récurrente quelques grands thèmes que nous efforcerons de cerner.

Après ces remarques préliminaires nous pouvons étudier les grandes questions qui concernent les agglomérations choisies et pour cela nous suivrons l'ordre des thèmes proposés ci-dessous.

\section{Les problèmes retenus dans les six agglomérations}

A travers notre prisme nous avons retenu plusieurs grands thèmes qui se retrouvent en général dans toutes les agglomérations étudiées.

Le premier concerne l'urbanisme avec des sous-thèmes tels que:

- aménagement urbain

- question du logement, question foncière et immobilière, le cadre bâti

- équipements urbains

Le deuxième est liée à la question des transports et des sous-thèmes suivants:

- transports publics

- circulation privée

- politique globale des transports réflexion transports publics/privés

infrastructures liées aux transports publics et à la circulation - parkings

\subsection{L'urbanisme}

L'urbanisme en tant que pratique de l'urbanisation est un tout global qui demande à être structuré et divisé en plusieurs sous-thèmes.

- aménagement urbain

- question du logement, pression foncière

- équipements urbains

\subsubsection{L'aménagement urbain}

Les villes devant la conjugaison de la reprise économique depuis les années 1980 et leur volonté de redynamiser leur image de marque, se lancent dans de grosses opérations d'aménagement ou de réaménagement urbain. Ces opérations peuvent s'inscrire dans le tissu central Espacitè à CF, le Rôtillon à Lausanne et Sulzer à Winterthur - ou péricentral - Europont à CF, Plainpalais, Wilson et Sécheron à Genève, Riponne-Tunnel et Flon à Lausanne, quartier de la gare à Neuchâtel. 
On peut noter que ces aménagements lourds concernent surtout l'espace péricentral et cela peut s'expliquer par plusieurs facteurs. Les centres et en particulier les hypercentres connaissent une situation qui a tendance à se geler, car ils font l'objet de règlements de protection draconiens, de densification considérable et d'une rente foncière très élevée qui freinent la mise en place de grosses opérations identiques à celle de Confédération à Genève. Les seules opérations qui touchent l'hypercentre, s'inscrivent dans un contexte bien spécifique - dégradation poussée du tissu urbain non protégé ou de densification faible d'un îlot. Le Rôtillon à Lausanne entre dans la première catégorie; par contre Espacité et le centre commercial Migros de CF s'inscrivent dans le deuxième cas. Ceci nous interpelle, va-t-on immuablement vers une pérennisation des hypercentres qui rappellent le développement des centres historiques? Cette option ne semble pas souhaitable car c'est une fossilisation d'une partie des villes et qui représente un danger à terme avec le déplacement de l'épicentre économique des agglomérations vers d'autres espaces. La situation socio-économique de CF et la volonté politique de constituer un véritable centre-ville image symbolique très forte de la ville - permettent de comprendre la réalisation de deux opérations urbanistiques dans le centre même.

La zone péricentrale a la faveur des grands projets. Il peut s'agir d'un projet global ou partiel de restructuration urbanistique. Le projet global déborde de son espace en modifiant un secteur considérable de la ville - Sulzer sur $114000 \mathrm{~m}^{2}$ à Winterthur qui vise à créer un nouveau centre à côté du centre actuel, Europont à CF constitue un projet de $20000 \mathrm{~m}^{2}$, trait d'union entre la ville et la «non ville» située endeçà des infrastructures ferroviaires, le Flon et l'implantation d'un nouveau pôle de développement de Lausanne au centre du futur réseau de transport en commun et l'opération liée à la remodélisation et à la restructuration de la place de la gare de Neuchâtel ont des implications qui dépassent de loin leur cadre. L'ensemble de ces projets comme celui de Sécheron à Genève qui est en cours de discussion, ne redynamiserait pas uniquement un îlot, voire un secteur urbain mais l'ensemble de l'agglomération par leurs retombées au niveau soit d'une extension du centre - Flon à Lausanne, Europont à CF -, soit d'une excroissance du centre - terrains Sulzer à Winterthur, Sécheron à Genève en relation avec la création d'un RER et l'implantation d'un pôle tertiaire, lié à la présence d'institutions internationales. La logique qui préside à la réalisation de ces opérations, au-delà de l'aspect économique considérable, est de redonner à la ville-centre, à son centre au sens large et à l'agglomération une nouvelle image de marque tant sur le plan urbanistique que sur le plan symbolique et social affirmant la volonté locale de relever les challenges urbains et donc socioéconomiques de notre monde. On peut ajouter qu'à côté de cette nouvelle fonctionnalité d'un espace précieux et fort convoité, il s'agit d'imprimer dans le vécu de la population de nouvelles valeurs qui lui permettent d'adhérer aux choix urbanistiques des autorités locales et d'intégrer une idéologie sociale consensuelle autour de nou- veaux modèles urbains de production et de consommation qui se marquent d'une façon très preignante à travers ces opérations de restructuration urbaine.

Ces projets de remodelage urbain profitent d'une condition extrêmement favorable, à savoir ils se situent dans les friches urbaines. Celles-ci sont liées à la présence de zones industrielles, artisanales de conception désuette selon nos normes modernes, à proximité des centres urbains et souvent reliées à l'infrastructure ferroviaire. Le déclassement de ces zones au nom des déséconomies urbaines - enclavement dans le tissu bâti, réseau routier disproportionné aux besoins actuels, impossibilité de modernisation sur place et nuisances nombreuses - impose une délocalisation de ces entreprises sur des sites industriels prévus à cet effet en zone périurbaine. C'est donc une chance pour certaines agglomérations de posséder ce type de friches urbaines, car dans ce cas les problèmes sociaux induits par les opérations de rénovation/délogement de population ne se posent pas ou peu.

Toujours en zone péricentrale des opérations partielles ou ponctuelles de moindre envergure se préparent. Il s'agit pour Genève de Plainpalais et de Wilson, pour Lausanne de l'îlot Riponne-Tunnel, pour Neuchâtel du quartier de Vausseyon. Leur logique est différente des précédentes. Une fraction du cadre urbain se prête à une opération limitée dans l'espace et dans ses interactions avec le reste de l'agglomération. Souvent il s'agit d’opérations de prestige - à Genève construction de complexes hôteliers haut de gamme - mais aux retombées limitées pour la ville, cependant entraînant des modifications au niveau du quartier qui peuvent donner naissance à des mouvements d'opposition plus ou moins marqués. Quant à Berne et Winterthur, il faut souligner le caractère non prestigieux des différentes interventions et qui s'inscrivent avant tout dans une politique de renforcement des équipements lourds à vocation utilitaires. La situation bernoise est également intéressante par le nombre de projets retenus; il s'agit de la construction du stade de Wankdorf avec 1000 postes de travail programmées en souterrain, de l'achèvement du projet lié à l'aire BEA, de l'extension et de la rénovation du casino et enfin de la construction d'une usine d'incinération des ordures à Schlachthof. Pour Winterthur la construction d'une usine d'incinération des ordures est prévue à Oberwinterthur et l'édification d'un centre commercial important MMM est retenue à Seen.

Les conflits générés par ces interventions touchent aussi bien les grosses opérations que les plus petites. Ces oppositions à ces projets urbains se retrouvent aussi bien dans des villes de taille fort différente et ceci montre la présence d'un courant multiforme de rejet systématique des opérations qui remettent en cause un ordre urbain que certains souhaiteraient immuable.

Ces mouvements multiformes de contestation débouchent souvent sur des référendums locaux au sujet des projets contestés et leurs résultats varient d'un objet à un autre et surtout d'une ville à une autre.

Le projet de CF Espacité a été fortement critiqué avant d'être quelque peu modifié - changement de la couleur 
rouge de la tour pour une couleur plus neutre le gris - et il a trouvé gain de cause lors d'une votation populaire en 1990. Par contre les Lausannois n'ont permis qu'à un seul projet sur cinq d'être réalisé, il s'agit de l'ensemble commercial Migros à proximité de Bel-Air. Les projets plus ambitieux du Rôtillon et du Flon ont essuyé des échecs notoires et pourtant lors de leur présentation au public, peut-être trop «technique» et trop «feutrée», ils n'avaient soulevé aucune critique dans la population lausannoise. Ce n'est qu'à l'approche des décisions officielles qu'un mouvement de contestation s'est constitué et a fait capoter les desseins du pouvoir local. Ces décisions populaires négatives obligent l'actuelle municipalité à tergiverser et à mettre en place des moyens efficaces pour tenter d'imposer sa volonté en matière urbanistique - plan directeur, délégation aux grands projets et une politique d'information crédible...

A l'opposé le cas genevois est beaucoup plus nuancé dans les mouvements d'opposition aux projets urbanistiques. C'est ainsi que les opérations Wilson et Plainpalais ont reçu un aval positif lors de votations communales, il est vrai de justesse. Par contre un projet scolaire a été repoussé par le corps électoral de la ville.

Nous pouvons tirer quelques hypothèses de ces différents cas. Le problème lausannois est exemplaire. Plusieurs élements peuvent expliquer ces séries de rejet. Comme par exemple le manque de souplesse, de diplomatie et voire de démagogie des autorités locales qui n'ont pas pris en compte à leur juste valeur l'évolution socioculturelle générale qui oblige une modification des comportements de type paternaliste et autoritaire des politiciens locaux. La pugnacité des groupes d'opposants et peut-être la mobilisation des «Neinsager» sont à retenir également pour comprendre ces échecs.

Dans ce sens, Genève représente une ouverture des élus au sujet d'une gestion plus souple qui a permis à des projets fort contestés de passer. A CF nous pouvons faire une contastation similaire. Dans ces deux villes les autorités locales ont dû se battre pour ces projets et donc entamer le débat avec leurs électeurs/citadins. Ils sont descendus de leur piédestal, attitude qui a sans doute été déterminante dans les résultats.

A Berne le recours systématique au peuple est une donnée fondamentale que l'on ne retrouve pas dans les agglomérations romandes, puisque 150 objets furent l'occasion d'une votation, dont beaucoup touchent la question urbaine au sens large.

Face à ces résultats deux lignes se dessinent. Pour certains on doit réaménager les droits populaires du moins leur usage, en les restreignant; pour d'autres il importe de soigner les relations publiques et donc de se lancer dans des opérations d'informations pour associer les citadins aux projets et à l'image de leur ville.

Nous venons de voir les projets qui se localisent dans la zone centrale et surtout péricentrale, mais le city-druck touche également la banlieue et des zones de plus en plus éloignées. A Genève plusieurs opérations sont programmées à Plan-les-Ouates, Veyrier et au Petit Lancy. A Winterthur le projet MMM fait l'état de consultations. A Lau- sanne plusieurs projets concernent le nord de la ville. A Neuchâtel le depart de Suchard de Serrières laisse la porte ouverte à plusieurs possibilités de réaménagement. Il s'agit de décongestionner le centre au sens large du terme, en délocalisant des activités et en attirant de nouvelles sur des périmètres qui sont prévus à cet effet. A Genève, l'exiguïté du territoire cantonal et la réglementation en matière d'aménagement du territoire imposent une densification de l'espace périphérique par déclassement de zone à faible cœfficient d'occupation du sol - zone villas devient zone à petits collectifs, etc. Lausanne jouit d'une situation plus favorable puisqu'elle dispose de réserves foncières importantes situées à proximité d'infrastructures de transports - Blécherette et vélodrome non loin d'un échangeur autoroutier, des établissements horticoles desservis par le tram sud-ouest et par un échangeur autoroutier.

\subsubsection{La question du logement, du cadre bâti}

La question du logement transcende l'ensemble des agglomérations avec, il est vrai, une acuité moins vive à $\mathrm{CF}$ que dans nos autres cas d'études. Le logement est une question toujours aussi lancinante aujourd'hui qu'à la fin du second conflit mondial. En effet, même si l'on ne parle plus de crise du logement, il est frappant de constater que celle-ci suscite d'importants projets de construction et que certains indicateurs signalent la pérennité de ce phénomène comme par exemple le taux de logements vacants et les opérations de squaterisation.

En 1986, le taux de logements vacants pour différentes agglomérations indiquait déjà une situation fort tendue dans le parc immobilier, avec les valeurs suivantes pour Lausanne $0,60 \%$, pour Berne $0,59 \%$ et enfin pour Genève $0,23 \%$. Même si cet indicateur peut être critiqué, il n'en demeure pas moins qu'il révèle avec beaucoup de netteté la situation tendue qui prévaut dans ce secteur. Il est à noter qu'une fois de plus l'effet de taille est à prendre en compte au sujet de cette situation tendue sur le plan du logement. Le marché immobilier est beaucoup plus asséché à Genève qu'à $\mathrm{CF}$.

Il n'est qu'à suivre les différentes revues de presse pour noter l'acuité de cette question et pêle-mêle nous rencontrons des articles sur le manque de logements et en particulier de type social, sur les opérations de réhabilitation et leurs incidences socio-économiques, sur les squaterisations de logements vides et sur des options telles que la récupération des "friches urbaines" ou le changement d'affectation et/ou de coeficient d'occupation du sol. Que l'on parcoure la presse à Genève ou à Neuchâtel, malgré l'effet de taille nous tournons autour des mêmes préoccupations. Donc la presse au niveau des articles est un indicateur de premier choix, mais les rubriques des lecteurs et les annonces immobilières sont également fort parlantes. Car le manque de logements se traduit par une flambée des prix, même si cette dernière est imputable aussi en partie à la politique monétaire appliquée.

A côté de cette source d'informations, nous pourrions prendre l'ensemble des statistiques concernant ce sec- 
teur. Les offices de statistiques des cantons de Vaud et de Genève sont révélateurs de la poussée immobilière nombre de demandes de permis de construire, nombre de mise en chantier, nombre de logements vacants, nombre de mutations de propriétés immobilières et enfin évolution des prix da la construction immobilière et des prix de vente du $\mathrm{m}^{2}$. C'est ainsi qu'à Berne le prix moyen dans l'hypercentre au niveau de la Schauplatz était de 30000 fr. le $\mathrm{m}^{2}$ en 1988 . Un record était atteint sur la partie sud la Spitalgasse avec 56000 fr. en $1987 .^{6}$

Enfin sur le plan politique cette question est devenue récurrente depuis quelques années. C'est ainsi que la ville de Neuchâtel est tenue de réaliser 500 logements sociaux suite à une initiative populaire qui a été acceptée en 1984 et qui commence à s'inscrire dans la paysage urbain par la réalisation de plusieurs projets immobiliers sociaux. L'actuelle municipalité lausannoise - rose/verte - a fait de la construction de logements sociaux son cheval de bataille. Il serait plus juste de dire que c'est le PS qui devant tenir compte de son électorat propose du logement social comme panacée au mal-vivre de la population. Sur ce point il s'oppose à son allié de circonstance - les Verts -, qui eux ne tiennent pas à sacrifier les derniers espaces libres de la commune pour la construction de logements - aérodrome de la Blécherette, le golfe, ou après désaffection le cimetière de Bois-de-Vaux. Donc comme on le voit le logement devient un enjeu politique primordial.

Devant la pertinence de cette question, les milieux économiques investissent ce secteur porteur et ceci se matérialise par des projets et des réalisations plus ou moins notables selon les problèmes propres à chaque agglomération. On peut distinguer différents types d'intervention, par exemple des opérations de réhabilitation se situant souvent dans les centres ou dans les quartiers de la fin du XIXe ou du début du XXe siècle et des opérations de construction immobilière plus ou moins vaste suivant l'espace disponible où elles se localisent - tissu dense de la zone péricentrale, ou plus lâche de la périphérie. Au niveau des intervenants nous trouvons des promoteurs privés, des sociétés coopératives ou des organismes communaux; de plus en plus on relève des opérations conjointes qui soulignent une nouvelle stratégie où les capitaux privés ont besoin d'une certaine caution publique - cf. projets genevois et bernois.

Les opérations de réhabilitation touchent toutes les agglomérations retenues; c'est un marché fort porteur pour les constructeurs et un accélérateur des changements sociaux donc de la ségrégation sociale entre quartiers voire entre rues. Un cas est très intéressant à prendre en compte, pas tellement au niveau de la ségrégation sociale mais de l'importance des réhabilitations entreprises. La situation tant socio-économique qu'historique $\mathrm{CF}$ explique les efforts faits par la municipalité en matière de politique de réhabilitation. Ce cadre urbain unique en Suisse fait l'objet de mesures conservatoires notoires qui passent par une prise en charge partielle des frais de réhabilitation entrepris pour la remise en état des logements. Pourtant c'est un cas où le problème spéculatif n'est pas virulent car le marché immobilier offre un nombre im- portant d'appartements à loyer modéré. Cet aspect s'intègre dans une politique de pugnacité que mènent les autorités locales et les forces économiques afin que CF possède là un atout de premier choix pour attirer des entreprises de l'extérieur. Dans les autres agglomérations les opérations de réhabilitation transforment des secteurs toujours plus étendues - Les Eaux-Vives, Plainpalais, Carouge pour l'agglomération genevoise; la Borde, Chauderon pour Lausanne, etc. -, en les vidant de leur contenu social et économique d'origine.

Quant aux grosses opérations immobilières nous en trouvons dans les zones péricentrales projet de Villereuse et de Sécheron à Genève, de l'opération RiponneTunnel à Lausanne, des terrains Sulzer à Winterthur et les réalisations de l'Ecluse et des Sablons à Neuchâtel. Ces projets sont beaucoup plus complexes à mettre en place car ils se situent dans un périmètre fort convoité - à savoir à proximité des centres existant - et qui présentent des opportunités de rentabilisation économique maximale. Mais à côté de l'intérêt des promoteurs privés, d'autres volontés se manifestent pour récupérer ces terrains dont certains se trouvent sur des friches urbaines, pour $y$ construire du logement social ou divers équipements qui font défaut.

C'est dans ces zones péricentrales que se déroulent actuellement les gros enjeux tels que les différentes concurrences entre logements/activités tertiaires, logements libres/logements sociaux, logements/équipements publics, bureaux/équipements publics et intérêts publics. Dans l'avenir cette situation ne fera que s'amplifier en s'étendant sur d'autres secteurs de la ville.

D'autres opérations immobilières se mettent en place en périphérie dans la zone suburbaine voire périurbaine. Dans ce contexte-là on peut noter le projet du Brünnen à Berne où à côté des normes rigoureuses de planification sont imposées des exigences architecturales très poussées en matière architecturale - matériaux, aspect phonique, formes. Cette opération associe la ville de Berne à des promoteurs privés. A Genève on peut signaler les cas de Plan-les-Ouates et de Veyrier, à Lausanne la poursuite de projets immobiliers aux Bossons et la réflexion au sujet de la Blécherette et du parking du vélodrome et à Neuchâtel les Acacias.

Au niveau des agglomérations nous devons signaler la poursuite de la périurbanisation qui touche des communes de plus en plus éloignées du centre commun. La multiplication de ces micro-opérations couvre des portions de plus en plus vastes du territoire, induisant des modifications radicales de l'espace rural et surtout des réalités sociales qui remplacent les anciennes relations sociales fondées sur l'appartenance à une même communauté rurale. Cependant face à cette tendance lourde de destructuration de l'espace rural, on doit signaler les mesures qui sont prises pour préserver certaines fractions du territoire de la boulimie des promoteurs. En particulier Genève freine le mitage de son canton et ceci est dû à une volonté politique qui s'appuie sur des considérations économiques et écologisantes. D'autre part l'aménagement du territoire, des espaces communaux ne relève pas 
des communes qui n'ont un droit que consultatif en la matière mais du canton. Situation dénoncée par certains partis politiques de droite au nom d'un libéralisme tout puissant et qui risque de donner lieu dans quelques années à un référendum sur le plan cantonal pour redéfinir c'est-à-dire revoir à la baisse les prérogatives du canton, pour renforcer le rôle des communes.

Dans nos agglomérations les clivages socio-économiques liés aux politiques communales ont tendance à se renforcer ne serait-ce qu'avec les règlements d'urbanisme - hauteur et gabarit limité, interdiction des collectifs petits et grands, surface minimale des parcelles de construction - et l'envolée des prix du $\mathrm{m}^{2}$ constructible. Pourtant quelques communes se posent des questions quant à leur devenir de leur zone résidentielle-dortoir et des options de développement différent sont envisagées avec tout d'abord des activités productives dans les hightech ne remettant pas en cause une certaine qualité de vie et ensuite par une révision de leur plan d'occupation des sols leur permettant une densification avec la production d'habitat groupé, voire de petits collectifs pour capter une partie de la main-d'œuvre pendulaire travaillant dans ces communes. Cette option est défendue par la commune du Mont dans l'agglomération lausannoise.

Enfin le dernier aspect que l'on doit aborder est relatif aux problèmes des squats et aux mesures prises ou envisagées pour soit les éradiquer ou soit les intégrer d'une façon ou d'une autre et à travers cette question se pose la politique des villes par rapport à leurs marginaux.

\subsubsection{Les équipements urbains}

Les équipements lourds qui ont été pendant plusieurs décennies un des éléments moteurs de l'urbanisme, sont moins présents à l'heure actuelle dans les revendications de la population ou dans les programmes électoraux, car les plus pressants et les plus importants sont déjà en place. Cependant cela ne signifie pas qu'aucun équipement ne se met en place ou n'est pas prévu aujourd'hui. La nature des besoins en équipements a évolué, soit il s'agit toujours d'équipements lourds mais la plupart du temps liés au domaine des transports - que nous verrons plus loin -, soit des réalisations au coup par coup et souvent de dimension plus faible - équipements scolaires, crèches, salle de réunion etc.

Pour toutes les agglomérations étudiées, on est frappé par leur modestie. A Neuchâtel certains projets pour rattraper un retard ou imputable par exemple à la réalisation d'une infrastructure de première importance comme l'autoroute entraîne contrairement aux autres agglomérations des opérations considérables à l'échelle de Neuchâtel. C'est ainsi qu'à proximité de l'échangeur autoroutier de Vausseyon deux équipements importants s'installeront, il s'agit de la gendarmerie et des PTT. Un autre équipement qui peut être comparé à une décentralisation de l'administration fédérale est la venue des statistiques fédérales dans le complexe qui se dessine au niveau de la gare. A Berne et à Winterthur signalons les projets de construire des usines d'incinération des ordures ména- gères qui va également se poser à Lausanne dans un avenir proche. Dans nos agglomérations la gestion des déchets est un poids de plus en plus lourd; c'est ainsi que Lausanne est obligée de faire traiter une partie de ses ordures dans la région de Saint-Etienne en France! Les gros centres commerciaux qui se sont ouverts durant les deux dernières décennies couvrent grosso modo la demande, car un seul projet important est prévu à Winterthur MMM de Seen.

\subsection{Les transports}

Il peut sembler curieux que l'on s'intéresse à la question des transports dans un champ de recherche réservé au quartier, pourtant il n'en est rien. Car la politique des transports touche l'ensemble des territoires et par là même, elle irrigue non seulement l'intégralité des agglomérations et de l'espace géographique dans lequel elles se situent, mais également nos quartiers.

La question des transports offre deux entrées. La première concerne le rôle des transports à notre époque dans la stratégie et l'image que développent les agglomérations pour attirer des entreprises hautement qualifiées. La seconde relève de la politique de développement des quartiers, tant sur le plan économique que social. C'est en ayant à l'esprit cette complémentarité que nous analyserons les interrogations qui nous interpellent au sujet de ce thème.

Les transports publics connaissent un regain d'intérêt lié à la prise en compte des questions écologistes et de la congestion de plus en plus poussée des centres villes et de larges périmètres urbains. Ce constat débouchant sur des déséconomies de plus en plus notoires, oblige les autorités publiques à prendre des mesures et l'on s'achemine vers la mise en place de politique globale des transports qui seront basées sur des restrictions en matière de circulation privée au profit d'une politique axée sur les transports collectifs.

\subsubsection{La politique des transports publics}

Après bien des atermoiements et des différences considérables entre nos agglomérations, on se dirige vers une conception globale des transports, qui, par la force des choses, met l'accent sur les transports en commun.

Pourtant, il est à noter que la situation est en général nettement plus favorable dans les agglomérations alémaniques en matière de transports collectifs. Pour tenter d'expliquer cette opposition, il faut nous reporter à une division socio-culturelle qui coupe l'Europe en deux: d'un côté les pays latins où l'on a sacrifié les réseaux de transports collectifs de type tramway dans les années 50 au nom d'une liberté mal comprise, symbolisée par la montée en puissance de la motorisation privée; et de l'autre, une Europe germanique et centrale plus consciente, plus responsable d'un équilibre entre les différents modes de transports. Ce clivage se retrouve en Suisse où il est pa- 
tent au niveau des agglomérations choisies. Selon la région linguistique, on part donc de deux constats spécifiques, ce qui explique déjà en partie une acuité de ce problème fort dissemblable selon les agglomérations considérées.

Que ce soit à Winterthur et plus encore à Berne, on est frappé par la politique des transports qui assure une priorité de facto aux différents types de transports publics. Le centre ville y est en grande partie réservé aux lignes de tram et de bus; la coordination et la complémentarité efficace de l'ensemble des moyens de transport collectifs, particulièrement au niveau de la gare, sont patents. Le réseau très serré de tram innerve le centre et la zone péricentrale. Non seulement la qualité du service est remarquable (fréquence, confort, rapidité), mais encore la politique tarifaire est particulièrement incitatrice (abonnements).

La plupart de ces éléments positifs se sont mis en place assez facilement, car il existait déjà un réseau efficace de transports collectifs jouissant d'une image de marque valorisante. Les améliorations apportées au fil des ans ne devaient pas y surmonter un handicap aussi lourd que celui des agglomérations romandes. En définitive, cette politique des transports en commun ne représente pas à l'heure actuelle l'enjeu urbain le plus considérable.

L'existence d'un réseau global de transports en commun particulièrement efficace constitue un plus important dans le choix des stratégies des groupes économiques, tout en étant pour la population concernée un atout indéniable, non seulement dans sa fonction utilitariste, mais aussi de plus en plus dans sa fonction écologiste et son rôle symbolique. A ce niveau-là, on ne peut que remarquer les positions communes des représentants des milieux économiques et de ceux de groupes à l'idéologie opposée: les écologistes, une partie de la gauche et la marge. Ceci ne signifie pas pour autant qu'il n'y ait aucun problème. En effet se pose à Berne une question embarrassante: quel type de cohabitation peut-on mettre en place dans l'hypercentre entre les piétons et les trams. Les artères vidées de la circulation automobile font l'objet d'une large appropriation par les piétons et cela quelquefois au risque de leur vie. En ce moment, une réflexion est conduite autour de ces enjeux; réflexion qui est d'un ordre bien différent de celle qui se déroule dans les villes romandes où l'enjeu se situe entre voitures et transports collectifs. A une échelle bien différente, la même interrogation apparaît à Neuchâtel au sujet de l'éventuelle fermeture de la rue du Seyon aux transports collectifs.

Face à cette situation que l'on pourrait qualifier «d'idéale» selon le prisme romand, nous devons nous poser quelques questions sur les pesanteurs inhérentes aux cités francophones. En particulier, qu'en est-il d'une réflexion sur une politique globale des transports en commun et de ses premières retombées concrètes?

Il nous faut souligner de façon rituelle l'effet de taille qui joue aussi en ce domaine un rôle considérable. Mais aussi étonnant que cela puisse paraître, les autorités de La CF se posent de nombreuses questions sur leur réseau, qui s'inscrivent dans une même logique générale que l'on retrouve dans des agglomérations beaucoup plus peuplées. Les problèmes de détail, fréquence, rapidité, priorités, sont traités comme ceux d'une grande ville et nous pouvons percevoir la politique volontariste globale menée par La CF pour dépasser des inconvénients liés au contexte tant géographique que socio-économique de leur ville.

Pour les autres agglomérations romandes, il est à remarquer que par l'effet soit de réalisation d'infrastructures autoroutières notoires - autoroute de contournement de Genève, construction de la traversée autoroutière de l'agglomération neuchâteloise - soit de décisions populaires - référendum demandant la mise en place d'une politique globale des transports à Genève, initiative refusée de peu en 1989 à Neuchâtel pour des transports publics efficaces et bon marché - soit par la construction fort avancée d'une ligne de tram à Lausanne, la situation est en train d'évoluer très rapidement.

Le retard accumulé par ces agglomérations par rapport à celles de Berne et Winterthur ne sera pas comblé par ces mesures. Toutefois, celles-ci indiquent qu'enfin une volonté politique se dessine pour sortir de l'ornière suicidaire du tout auto suivie globalement jusqu'à présent depuis le démantèlement des réseaux de tram qui existaient encore au début des années 50 dans ces agglomérations. Ces politiques de développement des transports collectifs s'inscrivent dans des réflexions globales sur les transports en général, donc en y intégrant les questions relatives au transport privé. Cette globalisation des problèmes est impérative pour plusieurs raisons. On ne peut pas du jour au lendemain interdire de facto les centres villes aux automobiles puisque jusqu'à une date toute récente on les a incité à venir dans le centre, ne serait-ce que par la construction d'imposants parkings centraux. Pourtant l'asphyxie des centres au sens large du terme et d'autres portions des agglomérations, surtout à certaines heures, impose la mise en place de mesures coercitives qui ne peuvent être prises qu'en échange d'une amélioration très nette des réseaux de transports publics existants. Il ne s'agit plus de bricoler des mesures ponctuelles et partielles - couloirs réservés aux TP, priorités aux carrefours... - qui très vite sont dépassées par l'augmentation générale du trafic et qui induisent aussi bien le mécontentement des usagers des TP, qui se rendent bien compte de l'inefficacité de ces innovations minimes, que des automobilistes-électeurs qui perçoivent la dégradation de la circulation comme étant une conséquence des faveurs faites aux TP.

La conscientisation dans les agglomérations de Suisse romande s'est faite petit à petit depuis une vingtaine d'années. Les écologistes pour des raisons relatives à la pollution et la gauche pour des raisons imputables à la dichotomie transports public/transports privés renvoyant à un débat de société très vif à l'époque, se sont efforcés de populariser cette problématique à un moment où le tout voiture triomphait largement. Les chocs pétroliers, par leur répercussion sur le porte-monnaie des automobilistes et la menace d'une aggravation de la pollution ont 
permis d'accélérer cette prise de conscience dans le public.

A l'heure actuelle, même s'il existe toujours des clivages idéologiques entre les deux types de transports, ceux-ci ont tendance à perdre de leur prégnance avec la dépolitisation partielle du problème qui se déplace sur le plan écologique, sur le plan technique et donc dans la sphère de l'économie.

Nous pouvons souligner qu'actuellement les projets et les réalisations qui se mettent en place annoncent une inversibilité des mesures prises en faveur des transports en commun: MESO et allongement du LEB à Lausanne, projet de la ligne de tram 13 à Genève entre Sécheron, la gare et Carouge... Cependant, l'échéancier de ces mesures est encore globalement très flou, quelle que soit l'agglomération étudiée. Et ceci, d'autant plus qu'il faut garder à l'esprit certaines opérations qui constituent de véritables serpents de mer: prolongement du LausanneOuchy en direction de la Blécherette ou du CHUV...

Le référendum obligeant les autorités genevoises à présenter pour 1992 une étude globale des transports est symptomatique de la dénonciation par le corps électoral d'un laisser-faire en matière de transports cette votation populaire impose aux autorités de prendre leurs responsabilités. Dorénavant, celles-ci ont en contre-partie un aval populaire qui ne peut que les aider à affronter le lobby des automobilistes.

La situation des TP lausannois est à peu près identique à celle des genevois, avec une nuance de taille: des mesures y sont prises pour favoriser les TP. Genève, qui jouit d'une ligne de tram à doubles voies et en site propre, la plupart du temps, reliant Carouge à Moillesulaz en desservant l'hypercentre, possède un atout considérable par rapport à Lausanne. Toutefois, Genève hésite à mettre en place un réseau de TP à partir de cette véritable colonne vertébrale. Lausanne réalise une ligne de tram MESO (métro sud-ouest) qui part du centre, dessert l'université et I'EPFL ainsi que de grands ensembles, avant de rejoindre la gare de Renens qui verra ainsi son rôle grandir. Cette première liaison audacieuse pèche cependant par le parti pris retenu, à savoir la voie unique qui n'offre pas la même capacité de transport que la voie double et qui surtout peut être totalement paralysée par un incident quelconque. Saluons cette première réalisation qui va en entraîner d'autres, dont la plus importante sera la construction d'un pôle central de correspondances au centre ville, en relation avec la gare, Ouchy, les communes desservies par le LEB (Lausanne-Echallens-Bercher) et par la suite avec d'autres secteurs de l'agglomération.

A Genève, les événements dépendent non seulement de l'étude de 1992 mais aussi de décisions qui sont liées à la réalisation d'un RER et à la LGV, ligne à grande vitesse, permettant de raccorder la ville au réseau de LGV français. Il s'agit dans ce cas d'imbriquer les transports publics et les transports ferroviaires. La construction d'un réseau RER dont Genève serait la plaque tournante engloberait une région dépassant non seulement les frontières cantonales en direction de Nyon, voire Lausanne, mais également la frontière nationale en direction
d'Evian, d'Annecy et de Bellegarde exigeant la construction de tronçons ferroviaires manquant, en particulier La Praille-Les Eaux-Vives. La LGV exigerait également une interconnexion entre Genève et le Genevois français. La promotion de Genève sur le plan des LGV européens implique donc immanquablement une modernisation des transports publics genevois. Quoi qu'il en soit, même si la LGV se fait attendre, la situation catastrophique en matière de transports de l'agglomération impose des solutions rapides et radicales. Le débat se situe au niveau de l'opposition tram/métro ou plus exactement tram/VAL (véhicule autonome léger) plus proche du tram que du métro. Le premier est un moyen de transport classique et donc parfaitement rodé, alors que le second, au coût global nettement plus élevé, est plus futuriste.

Quant à l'ouverture de l'autoroute urbaine à Neuchâtel, on peut se demander s'il ne faudrait pas profiter de cette occasion pour poursuivre la ligne de tram du Littoral en direction de Marin en récupérant une partie de la chaussé largement dimensionnée. En l'état actuel, aucun débat n'est lancé à ce sujet, ce qui est bien dommage car la ville, en se lançant dans une opération pilote, pourrait redéfinir une politique globale des transports, d'autant plus qu'elle s'intéresse à la politique de modération du trafic. Tous ces projets soulèvent une grosse interrogation: quel en sera le coût et quels en seront les moyens financiers? Ceci à un moment où les agglomérations traversent des temps difficiles au niveau de leurs finances.

Pour clore cette présentation, retenons que deux situation s'opposent entre les agglomérations alémaniques et romandes et que deux logiques sont en présence. L'une vise une fonctionnalité des transports, l'autre tend à imposer une image de marque avec l'introduction du VAL au détriment du tramway.

\subsubsection{Les transports privés}

L'asphyxie des villes provoque des mouvements d'opposition très nets contre l'utilisation immodérée de l'automobile - écologistes, AST... - mais en même temps les défenseurs de l'auto se regroupent en parti des automobilistes qui entrent dans la scène politique locale afin de faire reculer les mesures qui sont prises contre eux. L'enjeu est donc de taille. Les programmes se poursuivent au sujet des infrastructures routières - autoroutes, routes de contournement, parkings... - mais en même temps des stratégies sont élaborées pour enrayer cette marée de tôle: modération du trafic, refoulement des autos de certains périmètres, zones résidentielles...

La construction d'infrastructures lourdes se poursuit: autoroute urbaine de Neuchâtel empruntant deux tunnels, autoroute de contournement de Genève devant faciliter les relations entre l'autoroute du lac, l'agglomération franco-genevoise et l'autoroute blanche. Même si ces deux nouveaux tronçons visent la circulation de transit, ils s'apparentent à deux autoroutes urbaines. Ils devraient permettre une meilleure fluidité de la circulation à l'intérieur des agglomérations, par captage d'une importante partie du trafic local. 
A côté de ces infrastructures autoroutières d'autres opérations de contournement se mettent en place ou font l'objet de discussion, voire de votations négatives. L'autoroute de contournement de Lausanne n'étant pas suffisante pour freiner le trafic dans les zones périphériques de l'ouest et du nord en pleine expansion, une route de contournement desservant ces nouvelles zones est en train de se mettre en place, du moins sur les plans. Cette réalisation est néanmoins fortement contestée car elle augmenterait globalement les nuisances tout en les déplaçant. C'est ainsi que la route de contournement de Prilly a été refusée plusieurs fois ces dernières années lors de votations. Dans le même ordre d'idée, la route de contournement du Mont divise la population de la commune depuis plus de 10 ans. Paradoxalement, il semblerait que ce soit ce type de réalisations qui soulève le plus d'opposition car l'absence d'un maillon autoroutier apparaît comme une aberration sur le plan économique et sur celui de la qualité de vie. Les autoroutes qui s'arrêtent aux portes des agglomérations induisent un ensemble de difficultés cumulatives de moins en moins tolérées par leurs habitants. Par contre, lorsqu'il s'agit de doubler une voie existante très surchargée, l'avantage global est beaucoup moins net que dans le cas précédent.

Au Mont, la route de contournement en projet borderait des quartiers récents et couperait des propriétés agricoles. La conjonction des intérêts agricoles et ceux liés à la qualité de la vie des résidents bloque le projet et instaure une coupure entre quartiers déjà touchés par le trafic et ceux qui ne le sont pas (encore) d'une part, et d'autre part entre agriculteurs aux stratégies différentes selon leurs projets: maintien de l'exploitation, lotissement de leurs terres...

Les enjeux au niveau de la construction des infrastructures routières ne se situent pas tant au niveau des grosses opérations - autoroutes - qu'à celui d'operations moins marquantes mais plus nombreuses et qui ont tendance, les unes ajoutées aux autres à surimposer un nouveau réseau routier dans des zones en pleine expansion, qu'elles soient résidentielles, industrielles ou commerciales.

Nous retrouvons des projets de ce type aussi bien à La $\mathrm{CF}$, à Neuchâtel, à Lausanne, qu'à Berne et l'on pourrait encore placer la traversée de la rade à Genève dans ce contexte-là. Ce phénomène est inhérent au city-druck.

Le stationnement, véritable casse-tête pour les autorités, induit une série de mesures tantôt lourdes, tantôt légères. La poursuite de la construction de parkings au centre ville aussi bien à La CF (Espacité, Migros) qu'à Neuchâtel (Pury, gare, Balance) indique un rattrapage de ces deux villes par rapport aux autres agglomérations. Par contre, des parkings de dissuasion sont prévus dans les autres agglomérations à proximité des échangeurs autoroutiers (Lausanne Nord, Ouchy), ainsi qu'en zone péricentrale (Le Flon à Lausanne, Sécheron à Genève). Pour freiner le déplacement en automobiles privées des pendulaires, Berne a mis en place une politique de stationnement qui privilégie les résidents au détriment des banlieusards et des automobilistes étrangers à Berne. Cette politique n'est pas encore reprise dans les autres agglomerations.

Des mesures coercitives à l'encontre de l'automobile sont appliquées, comme par exemple la poursuite des fermetures (ou du moins l'interdiction de stationner) de certaines rues aux voitures (rue du Seyon, place du Coq d'Inde, esplanade de la Collégiale à Neuchâtel, rue Haldiman, axe central de première importance de l'hypercentre lausannois, restrictions dans la vieille ville de $\mathrm{Ge}-$ nève) ou la pose de gendarmes couchés, de goulets d'étranglement, de giratoires. Les opérations de piétonnisation sont aujourd'hui mieux acceptees que les premières expériences, car elles entraînent une nouvelle image de marque pour les rues concernées. Elles sont donc dans l'ensemble (il y a toujours des oppositions!) soutenues par les commerçants et les promoteurs immobiliers. Les milieux financiers se réapproprient les centres villes - quartier de la gare à Berne, rive droite à Genève - phénomène qui s'accompagne souvent d'une piétonnisation favorable au commerce.

Un dernier point qui se manifeste actuellement avec beaucoup de force est à souligner: la modération du trafic. Alors que certaines agglomérations alémaniques se sont déjà lancées dans ce type d'opération, Neuchâtel est en Suisse romande la seule agglomération à tester cette nouvelle politique (cf. étude de l'IREC). Un succès, même partiel, de ce projet pilote ne manquera pas d'intéresser les autres villes romandes. S'appuyant sur tout un train de mesures (zones résidentielles limitées à $30 \mathrm{~km} / \mathrm{h}$, recalibrage de la chaussée, gendarmes couchés, îlots de sécurité...) la politique de modération du trafic vise une réappropriation de certaines rues par des utilisateurs en sursis: enfants, personnes âgées, piétons, cyclistes... si Neuchâtel semble avoir pris une longueur d'avance sur toutes les autres agglomérations romandes, il faut souligner que ces dernières tâtonnent en ce domaine: Genève cherche à limiter la circulation pour lutter contre la pollution et l'insécurité, des quartiers lausannois réclament des mesures... Cette nouvelle politique de modération de la circulation offre aux habitants la possibilité d'une reprise en main de leur ville.

\subsubsection{La politique globale des transports}

La gestion économique d'une agglomération passe obligatoirement par la régulation des problèmes liés aux différents types de transports. Dans cette perspective globalisante, on ne peut plus s'occuper d'un mode de transport en lui-même, sans tenir compte des autres et de la circulation en général.

Cette vision apparaît nettement dans les agglomérations retenues, tout au moins au niveau de la réflexion. C'est ainsi qu'à Lausanne une strúcture efficace et transversale entre les différents services de la ville s'intitule «Délégation aux grands projets et aux transports» et intègre pleinement la totalité des modes de transport dans la problématique urbaine. La votation genevoise de 1989 demandant un projet global des transports pour 1992 en y incor- 
porant tous les modes de transport, la traversée de la rade et la restructuration du réseau des TPG va dans le même sens. Pour ces deux agglomérations, les retards accumulés en matière de politique globale des transports et leur acuité du fait de leur taille expliquent qu'elles soient véritablement les seules à se pencher sur ces problèmes. En effet, que ce soit à Berne, à La CF ou à Neuchâtel (projet de modération du trafic), l'urgence en la matière est moindre. Néanmoins la capitale fédérale étudie la prolongation d'une ligne suburbaine - Muri/Helvetiaplatz en direction de la Bahnhofplatz.

Cette politique globale, même si elle est acceptée ou réclamée par une partie de la population et les milieux économiques, ne va pas sans poser de nombreuses interrogations, car au nom d'un libéralisme économique désuet se met en place un pseudo parti des automobilistes de type poujadiste. Ce type d'opposition, qui est un combat d'arrière-garde, même s'il apparait folklorique, peut bloquer totalement et pour une durée indéterminée toute approche globale pour rendre les villes plus vivables.

\section{Conclusion}

A la fin du vingtième siècle, les agglomérations helvétiques connaissent des bouleversements qui tendent à les restructurer après une période de destructuration. $\mathrm{Ce}$ processus induit par une logique économique peut rencontrer les préoccupations de la population lorsqu'il s'agit de prendre en compte la dimension écologique qui devient un facteur de ségrégation sociale de plus en plus net entre quartiers d'une même agglomération.

Les problèmes, grosso modo, sont identiques entre nos agglomérations, par contre leur acuité et les réponses que l'on avance, peuvent être différentes pour certains objets
- place des transports en commun, rôle des partis ou des associations, question de la marginalité.

Plusieurs de ces enjeux contemporains doivent se traiter à l'échelle de l'agglomération qui doit voir ses pouvoirs grandir afin d'assumer ses responsabilités qui ne tiennent pas compte des limites communales souvent surannées pour faire face aux conséquences de l'urbanisation. Il est inconcevable de se pencher sur les effets du city-druck ou sur le développement d'un réseau de transport dans les limites étroites d'un périmètre politico-administratif hérité d'une époque révolue. Cette réflexion correspond aux débats qui auront lieu dans l'avenir pour redéfinir les pouvoirs dans nos agglomérations.

\section{Notes}

' II s'agit de Berne, La-Chaux-de-Fonds CF, Genève GE, Lausanne Lsne, Neuchâtel NE et de Winterthur WI.

${ }^{2}$ Bassand, M. Villes, régions et sociétés, Presses polytechniques romandes, Lausanne, 1974

Grosjean, G. Die Schweiz: Städte, Geographisches Institut der Universität, Bern, 1984.

OEPR/RORREP, Les enjeux de l'urbanisation: Agglomerationsprobleme in der Schweiz, Peter Lang, Berne, 1988. Racine, J-B. et Raffestin, C., Nouvelle géographie de la Suisse et des Suisses, Payot, Lausanne, 1990.

Rossi, A., La décentralisation urbaine en Suisse, Presses poIytechniques romandes, Lausanne, 1983.

${ }^{3}$ Merlin, P. Dictionnaire de l'urbanisme et de l'aménagement, PUF, Paris, 1988.

Busset, T. et Schuller, M. Du quartier à l'agglomération: système d'indicateurs et de découpages urbaines, FNRS, PNR 25, Zurich, 1989.

${ }^{4} \mathrm{cf}$. note 2, Dictionnaire de l'urbanisme et de l'aménagement.

${ }^{5} \mathrm{cf}$. note 1.

${ }^{6}$ Der Bund du 3.07.1990, Berne. 